\title{
Whispering-gallery modes and Purcell effect in a Si-nanocrystal-based single microdisk resonator
}

\author{
M. Ghulinyan ${ }^{1}$, D. Navarro-Urrios ${ }^{2}$, A. Pitanti ${ }^{2}$, A. Lui ${ }^{3}$, G. Pucker ${ }^{1}$, and L. Pavesi ${ }^{2}$ \\ ${ }^{1}$ Micro-Technology Laboratory, Fondazione Bruno Kessler, Via Sommarive 18, Povo I-38050 Trento, Italy \\ ${ }^{2}$ Nanoscience Laboratory, Dept. Physics, University of Trento, Via Sommarive 14, Povo, I-38050 Trento, Italy \\ ${ }^{3}$ Materials and Analytical Methods Laboratory for Biosensors and Bioelectronics, Fondazione Bruno Kessler, Trento, I-38050 \\ Trento, Italy
}

\begin{abstract}
We report on visible light WGM emission from Sinanocrystal-based all-active microdisk resonators. The observed significant, 13-fold mode narrowing (Q 2800) at lowest excitation powers is attributed to the attenuation of pump-induced excited carrier absorption loss mechanism.
\end{abstract}

\section{INTRODUCTION}

Monolithic resonators such as micro-disks, rings and toroids are attracting an intensive and rapidly evolving research. Such structures are characterized by so-called whispering-gallery modes (WGM) [1], which are circularly propagating optical modes suffering continuous total internal reflection inside the resonator. Microdisk resonators are extremely attractive both from device application and fundamental points of view [2]. Optically passive microdisks, based on transparent materials with negligible absorption losses, lead to extremely high quality factors $\left(\mathrm{Q} \sim 10^{6}-10^{10}\right)$, while active resonator systems, such as III-V semiconductor quantum dot microdisk lasers, report active Q's of $10^{3}$ to $10^{4}$ in the visible and near infrared wavelength range $[5,6]$.

The recent challenges in silicon photonics towards using nanocrystalline $\mathrm{Si}(\mathrm{nc}-\mathrm{Si}$ ) as an integrated light source have boosted an intensive research in the last decade [7-9]. However, as an important cavity system, nc-Si-based microdisk structures have been little studied and only few works appear in the literature [10]. In Ref.[10] quality factors of few hundreds have been reported for microdisk arrays of nc-Si $/ \mathrm{SiO}_{2}$ superlattices.

Here we study the WGM emission properties of single microdisk resonators with an optically active disk material made of luminescent nc-Si embedded in SiOx matrix. We report on sub-nanometer WGM resonances corresponding to quality factors as high as 2800 around the wavelength of 800 $\mathrm{nm}$, which to our knowledge are the highest among the previously reported values in nc-Si-based systems. We demonstrate the importance of exciting a single resonator out of the mass-produced microdisk array in order to reveal the fine modal structure in the light emission. Additionally, we show an almost 13-fold narrowing of characteristic linewidths at lowest excitation power associated to an attenuation of excited carrier absorption losses. Finally, we give first experimental evidences on a possible quantification of Purcell enhancement of nc-Si spontaneous emission through time-resolved PL measurements.

\section{EXPERIMENTAL}

Our samples have been produced using standard silicon micro-fabrication technology. The disk material has been realized through plasma enhanced chemical vapour deposition (PECVD) of $135 \mathrm{~nm}$ of $\mathrm{Si}$ rich silicon oxide (SRO) on top of crystalline silicon wafers. A successive one hour annealing in an $\mathrm{N}_{2}$ atmosphere at $1100^{\circ} \mathrm{C}$ leads to the formation of $\mathrm{Si}$ nanocrystals in the $\mathrm{SiOx}$ host. Then the wafers were photolithographically patterned and dry etched anisotropically to form arrays of microdisk structures with diameters ranging from $2 \mu \mathrm{m}$ to $10 \mu \mathrm{m}$. The crystalline wafer has been wet-etched isotropically to form the mushroom-like microdisks (Fig. 1(a)).

We performed room temperature micro-PL measurements using the $488 \mathrm{~nm}$ line of an Argon laser. To observe the characteristic WGM emission, the PL signal was collected and analyzed in the plane of the microdisks (more details in Ref. [11]).

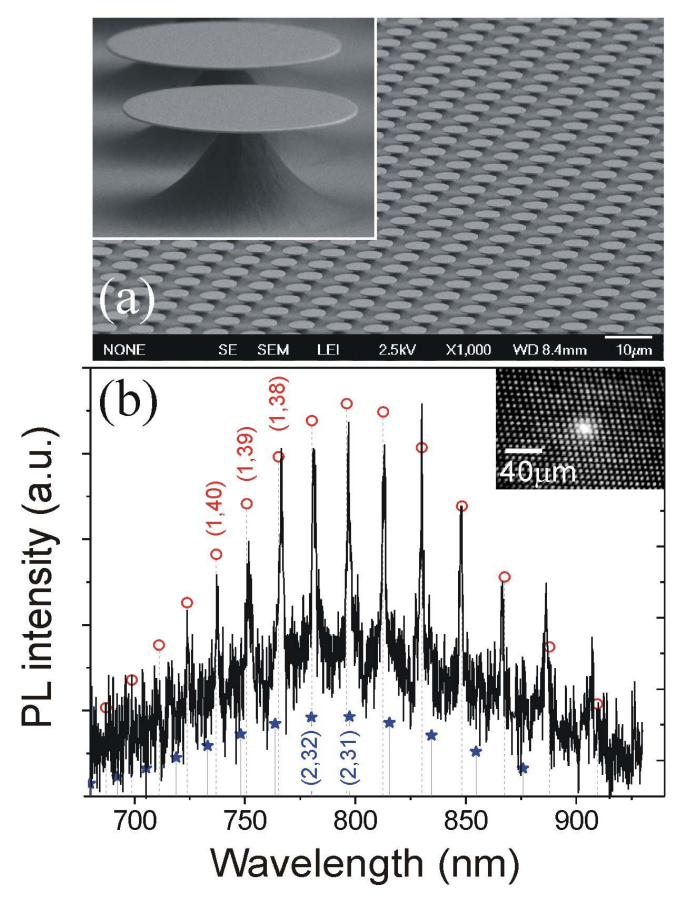

Figure 1. (a) Scanning electron microscope images of the array and the single disk resonator. (b) Measured TE-polarized WGM spectrum of an $8 \mu \mathrm{m}$ diameter microdisk is plotted together with the simulated peak positions for the first radial mode family (0). (Inset) The bright spot in the photograph is the direct image of the visible PL emission of nc-Si from a single disk resonator.

\section{RESUlTS AND Discussion}

We focused the excitation onto an individual microdisk and recorded the resulting PL emission. In fact, from Fig. 1(b) one observes immediately the fine WGM structure of the single microdisk. We observe sub-nanometer emission lines, corresponding to quality factors of almost $3 \times 10^{3}$, limited by the spectral resolution of our micro-PL setup (higher precision measurements may reveal higher $\mathrm{Q}$ values). Both 3D finite difference time-domain simulations and experiments confirm that such thin microdisks do not support guided transverse-magnetic (TM) modes because of the very low effective index for this polarization $\left(\mathrm{n}_{\mathrm{eff}}=1.08\right)$.- Thus, all the observed spectral peaks are TE-polarized and belong to the same radial family, with corresponding azimuthal mode numbers extending from $\mathrm{m}=42(710.5 \mathrm{~nm})$ to $\mathrm{m}=29(928 \mathrm{~nm})$ and an average mode spacing of $\sim 15 \mathrm{~nm}$. 
Below, we address some issues related to the influence of pumping power on the WGM characteristics of our microdisks, in particular, the observed significant linewidth modification. Figure 2 reports the measured Q's of few distinct resonances at $\lambda=754 \mathrm{~nm}, 768 \mathrm{~nm}$ and $849 \mathrm{~nm}(\mathrm{~m}=39$, 38 and 33 , respectively) of thin microdisks. We observe a monotonic ( 13-fold) decrease of $\mathrm{Q}$ factors as the pump power increases from 1.25 to $100 \mathrm{~mW}$. Such an impressive result needs a special attention; therefore, below we address the possible underlying mechanism.

The attenuating $Q$ factors suggest that at higher excitation powers we either introduce an additional loss source or enhance the existing ones. In a microdisk resonator, the total loss, resulting from different loss mechanisms, is expressed through the sum of inverse of possible limiting Q factors:

$$
Q^{-1}=Q_{\text {rad }}^{-1}+Q_{m a t}^{-1}+Q_{s s c}^{-1}+Q_{s a}^{-1}
$$

where the inverse of $\mathrm{Q}_{\mathrm{rad}}, \mathrm{Q}_{\mathrm{mat}}, \mathrm{Q}_{\mathrm{ssc}}$ and $\mathrm{Q}_{\mathrm{sa}}$ correspond to radiation, material (bulk absorption and propagation), surface scattering and surface absorption losses, respectively. In our case, the last two terms can be considered independent on the pump power within a good approximation. Thus, we focus on the remaining terms $\mathrm{Q}_{\mathrm{rad}}$ and $\mathrm{Q}_{\mathrm{mat}}$ which can be possibly modified by the excitation power. $\mathrm{Q}_{\mathrm{rad}}$ is related to the disk geometry, while the second one is calculated as $Q_{\text {mat }}=2 \pi n_{\text {eff }} / \alpha$, with $\alpha$ being the material loss coefficient.

First of all, from the comparison of WGM spectra measured at lowest and high pump powers no relative spectral shift of resonances neither modification of mode spacing is observed. Therefore, we exclude the possibility of changes in the effective indices or the disk sizes due to thermal heating effects. These observations lead us to suggest that the power-dependent $\mathrm{Q}$ can arise from variations in $\alpha$ within the nanocrystal-based disk material.

In recent studies on nc-Si containing waveguide systems, pump power induced losses at $1.5 \mu \mathrm{m}$ wavelength have been addressed and attributed to excited carrier absorption (ECA) [12]. We believe that the same mechanism occurs here; $\mathrm{Si}$ nanocrystals absorb strongly at visible wavelengths creating an exciton. Part of the excitons can successively absorb other photons to promote electrons to higher energetic levels in the nanocrystal conduction band. Such absorption events will enhance the cavity losses, causing the observed WGM broadening.

The ECA is proportional to the number of created excitons, N. At low pump powers, $\mathrm{P}$, when the number of excited nanocrystals is low, the ECA loss increases linearly with power. However, at high pump powers different phenomena can deviate this simple relationship; firstly, the number of non-excited nanocrystalls can not be considered any more independent on pump power. On the other hand, a number of processes, that influence exciton lifetime, such as Auger recombination and two-photon absorption, will induce additional non-linear $\mathrm{N}(\mathrm{P})$ behaviour.

The situation gets more complex when due to the cavity effect the spontaneous emission signal gets strong enough to affect the exciton population, as in the case of stimulated emission. When this becomes the dominant mechanism influencing the system loss, one expects that the absorption grows sub-linearly with power (increasing transparency), leading to an inversion in the tendency of the $\mathrm{Q}(\mathrm{P})$ curve (mode narrowing at high powers); hence, it would be possible to achieve net gain and eventual lasing at higher pump powers.

Thus, the possible presence of different loss mechanisms result in the observed complex behaviour of $\mathrm{Q}(\mathrm{P})$. In particular, we observe from Fig. 2, that all Q(P) curves show a clear "shoulder" at increasing pump powers from 4 to $12 \mathrm{~mW}$. In other words, variations in power in this range seem do not influence the FWHM of peaks, which could suggest a possible competing with ECA mechanism. A confident model and detailed studies for quantification of these phenomena are currently in course.

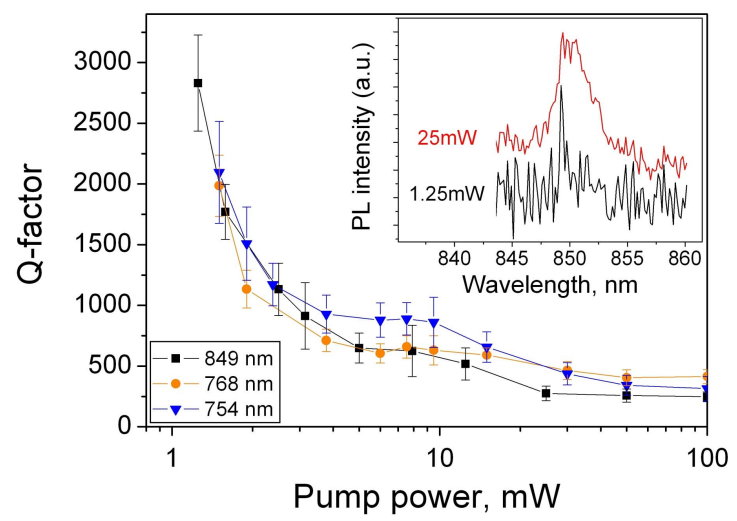

Figure 2. The measured Q-factors at increasing pump power are plotted at three different wavelengths, reporting an order of magnitude variation between two extreme pump powers. The inset shows the WGM mode at $\lambda=849 \mathrm{~nm}$ at the lowest and at a high pump powers.

We stress that ECA will figure as the main limiting factor for a possible multi-wavelength lasing from the nc-Sibased microdisk. We believe that the observed Q-factors of few thousands will be further enhanced after the SRO material optimization. Even with low, while inhomogeneously broadened gain spectrum of nc-Si, microdisk resonators with similar Q's could hopefully allow for a low-threshold laser action, in a similar way as in III-V semiconductor microdisk devices.

\section{Purcell enhancement of Spontaneous Emission RATE}

In 1946 E.M. Purcell suggested the possibility to enhance the spontaneous emission rate of an emitter in an environment with a high density of photonic states [13]. In the last years the same enhancement effect for an optical microcavity has been theoretically predicted and successively demonstrated in various high-quality resonators with III-V Quantum Dots as emitters [2, 14]. While almost all experiments on III-V semiconductors are being performed at low temperatures in order to maximally reduce the nonradiative recombination rate, we show here a first evidence of the Purcell enhancement for Si-nc in microdisks resonators at room temperature and a tentative estimation of the Purcell factor $F_{P}$.

Our measurements have been performed with micro-PL setup, using a mechanical chopper to modulate the laser pump. A monochromator coupled with a Photo-Multiplier Tube in the visible wavelength range was used to measure the Si-nc emission decay respectively at a peak and a valley of the WGM spectrum. The results are reported in Fig. 3.

In a semi-empirical model, one can consider the ratio of decay rates in a WGM peak $\left(\Gamma_{\text {tot,peak }}\right)$ and in a dip (bulk material, $\left.\Gamma_{\text {tot,dip }}\right)$, as

$$
\varepsilon=\frac{\Gamma_{\text {tot }, \text { peak }}}{\Gamma_{\text {tot }, \text { dip }}}=\frac{\Gamma_{\text {non-rad }}+F_{p} \Gamma_{\text {rad }}}{\Gamma_{\text {non-rad }}+\Gamma_{\text {rad }}},
$$


where $\Gamma_{\text {non-rad }}$ and $\Gamma_{\text {rad }}$ are the non-radiative and radiative decay rates, respectively. The ratio defined by Eq.(2) is a quantity which can be directly measured in experiment. The Purcell factor can thus be estimated through Eq.(2) in the following way:

$$
F_{p}=\frac{\tau_{\text {rad }}}{\tau_{\text {tot, dip }}}(\varepsilon-1)+1,
$$

where $\tau_{\text {rad }}$ and $\tau_{\text {tot,dip }}$ stand for the radiative and total lifetime of Si-nc, respectively.

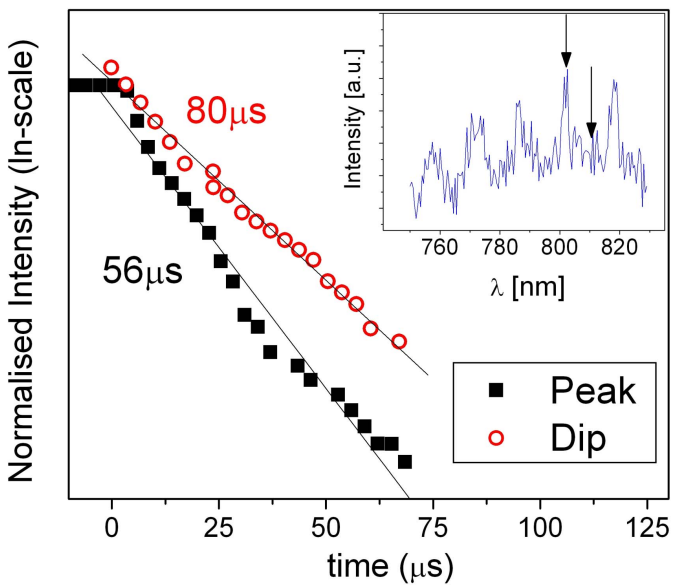

Figure 3. Time-resolved measurements of the Si-nc decay respectively in the peak $(802 \mathrm{~nm})$ and in the dip $(810 \mathrm{~nm})$ of the WGM spectrum. The low resolution WGM spectrum is shown in the inset, where arrows indicate the peak and dip wavelengths.

It is clear that $\varepsilon$ is a lower limit for the Purcell factor, and these coincide only in the limit of negligible nonradiative recombination rate. In the measurements reported in Fig. 3 we have found $\varepsilon=1.43$. In order to estimate the Purcell factor, one needs to measure in an independent way either the non-radiative or the radiative lifetime. Measurements on quantification of the real Purcell enhancement in Si-nc-based microdisk resonators are currently in course.

\section{CONCLUSIONS}

To conclude, we reported on PL emission properties of individual, optically active microdisk resonators with $\mathrm{Si}$ nanocrystals. We observed sub-nanometer whispering-gallery resonances in visible light emission with quality factors in excess of 2800 from single microdisk resonators. The influence of pumping power on the WGM narrowing has been addressed, showing more than an order of magnitude enhancement of Q-factors due to an attenuation of pumpinduced loss mechanisms. Moreover, we give first experimental evidences on quantification of Purcell enhancement through time-resolved PL measurements.

\section{ACKNOWLEDGEMENTS}

We acknowledge A. Picciotto and M. Wang for the help with PECVD deposition of wafers and L. Vanzetti for helpful discussions. This work has been partially supported by INTEL.

\section{REFERENCES}

[1] L. Rayleigh, "Further applications of Bessel's functions of high order to the whispering gallery and allied problems", Philos. Mag. 27, 100 (1914).

[2] K.J. Vahala, "Optical Microcavities", Nature (London) 424, 839 (2003).

[3] V.S. Ilchenko and A.B. Matsko, "Optical Resonators With Whispering-Gallery Modes-Part I: Basics", IEEE J. Sel. Top. Quantum Electron. 12, 76 (2006).

[4] A.M. Armani, D.K. Armani, B. Min, K.J. Vahala, and S.M. Spillane, "Ultra-high-Q microcavity operation in $\mathrm{H}_{2} \mathrm{O}$ and $\mathrm{D}_{2} \mathrm{O}$ ”, Appl. Phys. Lett. 87, 151118 (2005).

[5] Zh. Zhang et al., "Visible submicron microdisk lasers", Appl. Phys. Lett. 90, 111119 (2007)

[6] K. Srinivasan, A. Stintz, S. Krishna, and O. Painter, "Photoluminescence measurements of quantum-dot-containing semiconductor microdisk resonators using optical fiber taper waveguides", Phys. Rev. B 72, 205318 (2005).

[7] L. Pavesi, L. Dal Negro, C. Mazzoleni, G. Franzo, and F. Priolo, "Optical gain in silicon nanocrystals", Nature (London) 408, 440 (2000).

[8] Towards the First Silicon Laser, NATO Science Series, edited by L. Pavesi, S. Gaponenko, and L. Dal Negro (Kluwer, Dordrecht, 2003).

[9] A.S. Liu et al., "A high-speed silicon optical modulator based on a metal-oxide-semiconductor capacitor", Nature (London) 427, 615 (2004); H. Rong et al., "An all-silicon Raman laser", Nature (London) 433, 292 (2005).

[10] R.-J. Zhang, S.-Y. Seo, A.P. Milenin, M. Zacharias, and U Gösele, "Visible range whispering-gallery mode in microdisk array based on size-controlled Si nanocrystals", Appl. Phys. Lett. 88, 153120 (2006).

[11] M. Ghulinyan et al., submitted

[12] D. Navarro-Urrios et al., "Quantification of the carrier absorption losses in Si-nanocrystal rich rib waveguides at $1.54 \mu \mathrm{m} "$, Appl. Phys. Lett. 92, 051101 (2008).

[13] E. M. Purcell, "Spontaneous emission probability at radio frequencies", Phys. Rev. 69, 681 (1946).

[14] J. M. Gerard et al., "Enhanced spontaneous emission by quantum boxes in a monolithic optical microcavity", Phys. Rev. Lett. 81, 1110 (1998). 